\title{
Modeling Cosmic Ray Anisotropies at High-energy End of Solar Modulation
}

\author{
József Kóta*† \\ University of Arizona, Tucson, AZ 85721-0092, USA \\ E-mail: kota@lpl.arizona.edu \\ Kozai, Masayoshi \\ Shinshu University, Matsumoto, Japan \\ E-mail: kozai@u.shinshu.ac.jp \\ Munakata, Kazuoki \\ Shinshu University, Matsumoto, Japan \\ E-mail: kmuna@shinshu.ac.jp
}

\begin{abstract}
Solar modulation gives rise to intensity depletion and directional anisotropies in the distribution of galactic cosmic rays (GCRs). These anisotropies are observed to extend into the $100 \mathrm{GV}$ range, where the gyro-radius of GCRs becomes large and the robust diffusive equation of Parker is no longer applicable. We consider both diffusive and non-diffusive models to describe the high-rigidity regime of solar modulation. Our non-diffusive approach employs a model based on calculating energy losses suffered by GCRs along their trajectories through the heliosphere. We track GCR trajectories backward in time. A random magnetic field component is also added to account for scattering. The role of the wavy heliospheric current sheet (HCS) is studied in the two different polarity states of a 22-year magnetic cycle changing the tilt angle of HCS. Simulation results are compared with 44 years of observational data from the Muon Detector in Nagoya (Japan) and Neutron Monitors. We present preliminary simulation data to demonstrate general qualitative trends. Some implications of the simulation results are discussed.
\end{abstract}

35th International Cosmic Ray Conference - ICRC2017

10-20 July, 2017

Bexco, Busan, Korea

${ }^{*}$ Speaker. 


\section{Introduction}

The solar modulation of galactic cosmic rays (GCRs) extends into the $100 \mathrm{GV}$ range. The most prominent signatures of solar effects are the directional anisotropies which have been observed in ground-based measurements as small but consistent periodic diurnal variations on the rotating Earth. Remarkably, these observations [1], [2] gave the first sign of the apparent asymmetry between even and odd solar cycles: the phase of the "corotational anisotropy" of GCRs appeared to be earlier in one cycle than in the other one. This difference was later confirmed by a number of neutron monitors and muon telescopes. This puzzling phenomenon found a natural explanation in the regular drift motion of GCRs [3] and has been reproduced by numerical simulations considering particle trajectories [4] or the diffusive Parker equation including particle drifts [5],[6],[7].

The gyro-radius of 100GV GCRs is becoming comparable to characteristic scale of the heliosphere. GCRs at these rigidities are still considerably deflected by the spiral heliospheric magnetic field (HMF) but the Parker equation, which takes the limit of zero gyro-radius, becomes inaccurate. Particle motion is still sensitive to large-scale structures and is less so for small-scale fluctuations. Arguably the most prominent large-scale heliospheric structure is the wavy current sheet (HCS) co-rotating with the Sun. The tilt of the HCS, which changes during the solar cycle is an important driver of solar modulation and is expected to play profound role in producing anisotropies [4].

In this work we study the effect of the tilted HCS. We consider 44 years of anisotropy data from the Nagoya Muon Detector (MD) together with measurements of Neutron Monitors (NM). Figure 1 shows MD and NM have similar trends with distinct differences. Note the striking contrast between the magnitudes of the North-South anisotropies, $\xi_{N S}$ (bottom right) for the two measurements. The present work aims to identify general tendencies and is not intended for quantitative comparison.

Section 2 considers diffusive transport, which applies for up to a few tens of GV. In section 3, we discuss the method of tracking GCR trajectories and present a few illustrative examples.
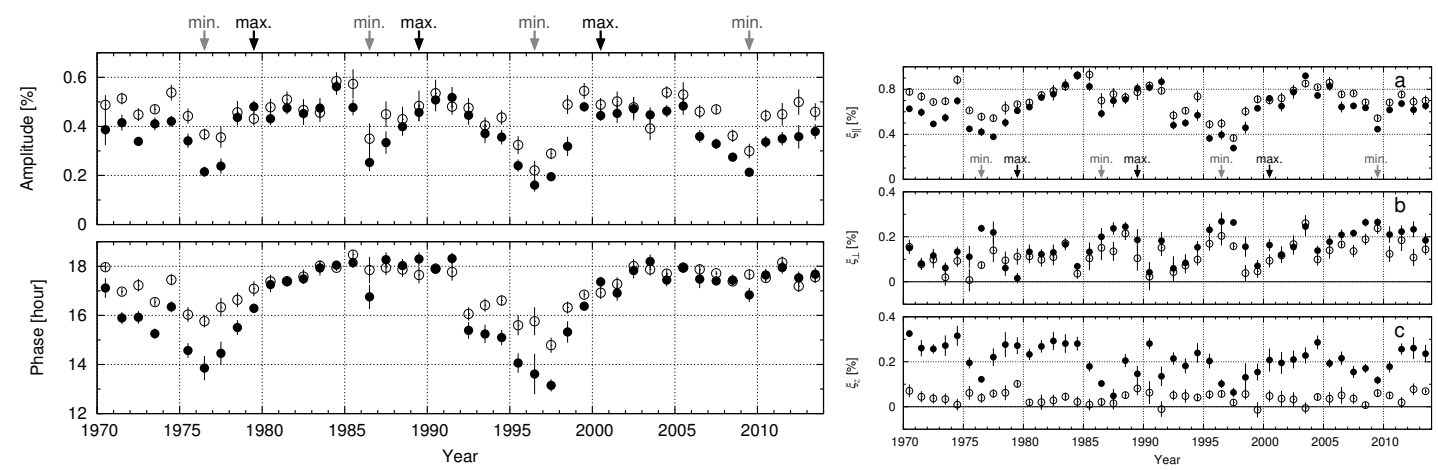

Figure 1: 44 years of GCR anisotropy data from the Nagoya Multi Directional Muon Detector (full circles) together with those from neutron monitors (NM). The median energies are $60 \mathrm{GV}$ for MD and $17 \mathrm{GV}$ for NMs, respectively. The left panel displays the amplitudes and phases of the yearly averages of the solar diurnal variation. The right panel shows the three components of the diffusive anisotropy, $\xi_{\|}, \xi_{\perp}$, and $\xi_{N S}$, after removing advection and Compton-Getting effect due to the orbital motion of the Earth. (from [8]) 


\section{Diffusive Transport: Parker Equation}

The diffusion tensor of the Parker-equation [9] consists of three different components: $\kappa_{\|}$ and $\kappa_{\perp}$ in the directions parallel and perpendicular to the mean magnetic field, together with an additional anti-symmetric component, that accounts for regular gyro-motion (often two different values of $\kappa_{\perp}$ are used for the two principal perpendicular directions). These all will conveniently be chosen to scale proportional to the local gyro-radius. Since modulation of GCRs above 10GV is small, it is only the ratios of $\kappa_{A} / \kappa_{\|}$and $\kappa_{\perp} / \kappa_{A}$ that count: an increase/decrease of all three elements would result in proportionally smaller/larger gradients, while the anisotropies would remain the same. A special case is the so called billiard-ball scattering which results from isotropic point-like scattering. Introducing the parameter $\beta=\left(\kappa_{\perp}^{2}+\kappa_{A}^{2}\right) /\left(\kappa_{\|} \kappa_{\perp}\right)$, we obtain $\beta=1$ for billiard ball, while the addition of the contribution of the random walk of field lines will increase $\kappa_{\perp}$ and yield $\beta<1$. Cases of $\beta>1$ tend to give 'unphysical" results such as reverse corotation.
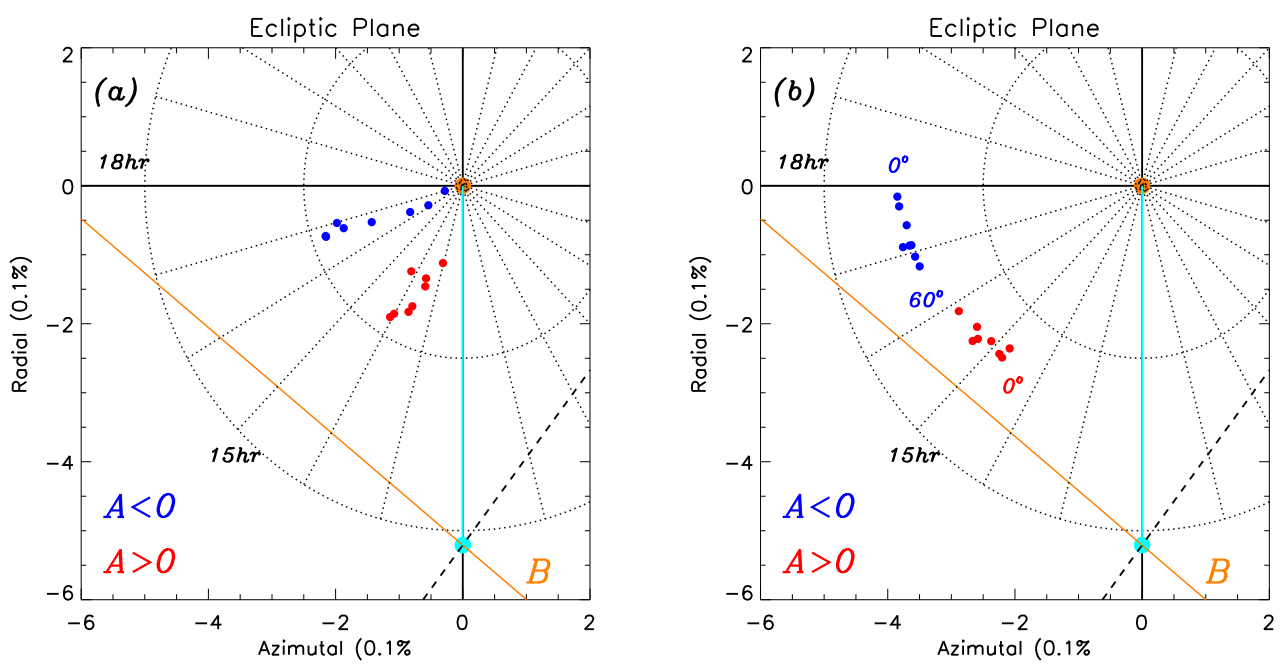

Figure 2: Numerical simulations for two different sets of diffusion coefficient (see text). The left panel considers "billiard ball" scattering. The right panel depicts a case of $\beta=0.2$. The phase difference between $A<0$ and $A>0$ is apparent. Orange lines mark the direction of the spiral HMF, the turquoise lines give the convective anisotropies, the diffusive components are to be measured from the turquoise dot.

Figure 2 and 3 show numerical results assuming an ideal Parker-spiral field with two different sets of diffusion coefficients, these are kept constant and only the tilt angle of the HCS, $\alpha$, is changed. The left panel takes billiard ball scattering $\left(\beta=1, \kappa_{A}=0.24 \kappa_{\|}\right)$. For this case the flat HCS does not give any azimuthal anisotropy. The anisotropy increases as $\alpha$ increases from zero to $\alpha=60^{\circ}$ for both $A<0$ and $A>0$. The phase for $A<0$, in this simulation, shifts to earlier hours partly because of the fast polar diffusion. Adding a random HMF component would result in phases closer to $18 \mathrm{hr}$ keeping the difference between the two polarities.

The right panels refer to a simulation with $\kappa_{A}=0.1 \kappa_{\|}, \kappa_{\perp}=\kappa_{A}$, hence $\beta=0.2$. For this case, there is a substantial azimuthal anisotropy for flat HCS, too. The amplitude of the solar diurnal vector is about the same for all tilt angles (from $0^{\circ}$ to $60^{\circ}$, the phases are separated by about 4 hours for flat HCS and approach each other with increasing tilt. The orange line indicates 

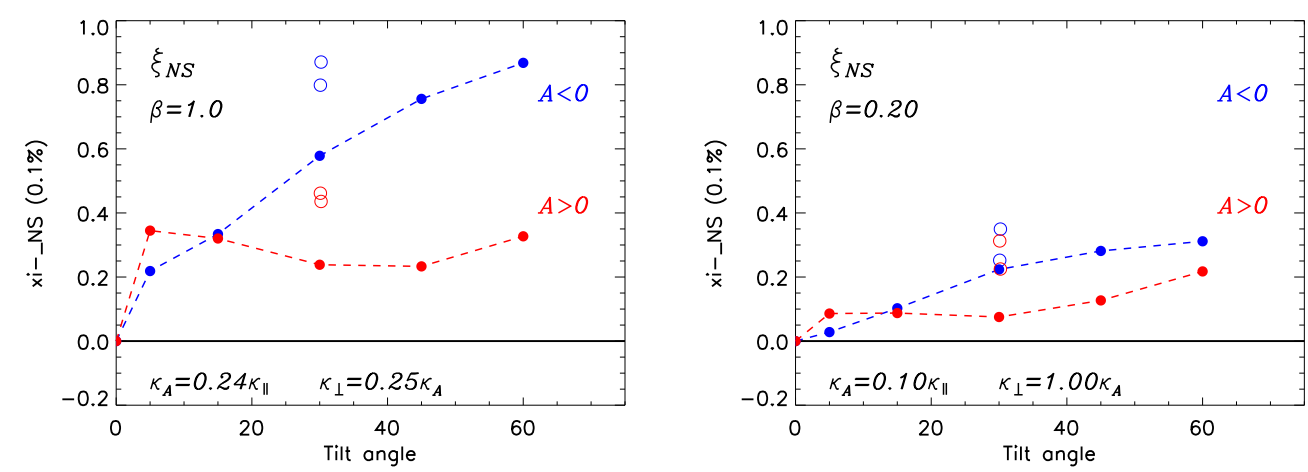

Figure 3: Tilt angle dependence of the North-South anisotropy, $\xi_{N S}$ for the two simulations discussed (see text). Open circles refer to configurations with more structured warped HCS (i.e. HMF with quadrupole moment).

the direction of the spiral field with the intent to guide the eye for determining the parallel $\left(\xi_{\|}\right)$ and perpendicular $\left(\xi_{\perp}\right)$ components of the diffusive anisotropy, these are to be measured from the turquoise dot (the turqoise vector accounts for advection). Inspection of Fig. 2 suggests that the effect of the tilt, $\alpha$, is more visible in the component $\xi_{\perp}$ in the left panel (billiard ball, $\beta=1$ ), while the parallel component, $\xi_{\|}$is more affected in the $\beta=0.2$ case (right panel).

The tilt angle dependence of the North-South anisotropy, $\xi_{N S}$, for the same simulations is seen in Fig. 3. $\xi_{N S}$ is increasing more or less linearly with increasing tilt angle for $A<0$, while it remains fairly small for $A>0$. The open circles refer to simulations when the tilt of the magnetic axis is $30^{\circ}$, but the HMF has a significant quadrupole moment (in other words, the HCS is not planar but warped near the Sun), so the maximum extension of sectored area is larger than $30^{\circ}$. This demonstrates that the tilt angle cannot be used as a single parameter, a more structured HCS is more effective in generating anisotropies. The maximum value of $\xi_{N S}$ does not reach $0.1 \%$ for either polarity in our diffusive simulations.

\section{Non-Diffusive Model}

GCRs are expected to be largely isotropic around the heliosphere. In the $100 \mathrm{GV}$ range, any small interstellar anisotropy is washed out, anisotropies seen at Earth reflect the different energy changes GCRs suffer along their different trajectories through the heliosphere. In this section we adopt an alternative non-diffusive model which is based on calculating energy losses/gains along GCR trajectories and which remains applicable at high rigidities. Trajectories are traced back from Earth in different initial directions spaced in 15 degree steps in declination and $1 \mathrm{hr}$ intervals in local time. The method is essentially identical with that of [4] except that, in addition to the standard corotating Parker spiral, the current model also allows a random component of the HMF. Hence trajectories are not deterministic but pseudo trajectories. Energy losses are averaged over a sample of 120 backward pseudo-trajectories. Intensity variations $\Delta J / J$ are connected to changes in energy, $E$, (or rigidity, $P$ ), using Liouville's theorem through the relation $\Delta J / J=\left(\gamma_{p}+2\right) \Delta P / P$, where $\gamma_{p}$ is the negative spectral exponent of the rigidity spectrum of GCRs. 
Regular motion in a fully steady state field would not introduce anisotropies (except for the shadow of the Moon or Sun). As an example, an idealized perfectly flat current sheet would not produce anisotropies. It is important, however, that this steady state condition ought to hold in the whole heliosphere including the heliosheath. Though this higly ideal state never occurs in reality, we can expect the anisotropies to decrease at solar minima when the HCS flattens. The corotating tilted HCS breaks the azimuthal symmetry and can produce anisotropies in the inner heliosphere. A prime example is the well known corotational anisotropy. In a strictly corotating field the the energy, $E$, and the angular momentum, $L_{i}$ are coupled, and the quantity $E-\Omega_{i} L_{i}$ is preserved. Frequent interaction with the sectored field and a great number of crossing the HCS can randomize the angular momentum at the exit of the backward trajectory, which will lead to a corotation in the inner heliosphere.
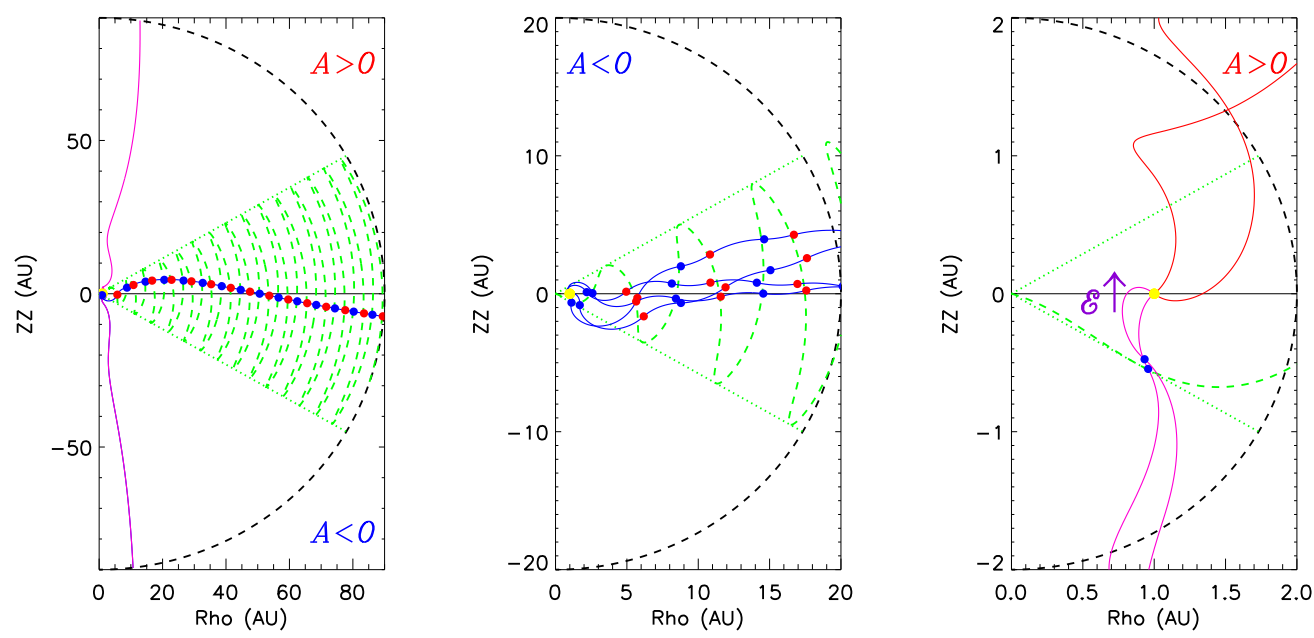

Figure 4: Illustration of typical backward pseudo-trajectories for $A<0$ and $A>0$ polarities. The magenta trajectories referring to $A>0$ move toward the poles, the blue trajectories $(A<0)$ are confined to the sectored area between heliolatitudes $-30^{\circ}$ and $+30^{\circ}$ (left). The middle and right panels zoom on $A<0$ and $A>0$ trajectories, respectively.

Figure 4 shows some 'typical' trajectories for the $A<0$ and $A>0$ polarity states of the Sun. For $A<0$, trajectories are largely confined to the sectored region of the HMF implying many sector crossings. The $A>0$ trajectories, on the other hand, quickly move to one of the polar directions and cross the HCS only a few times or not at all. This explains some of the peculiarities of the anisotropies in the $A>$ cycles. We note, that, for $A>0$, the $V \times B$ electric field points away form the HCS in the poleward directions. GCRs coming from either pole have to move against the electric field and lose energy. In some fortunate cases, however, GCRs move in the direction of the electric field and can gain energy between their last sector crossing (i.e. first crossing backward) and arrival at Earth. These will be the preferential viewing directions where intensity maxima can be expected.

We present numerical simulations for $100 \mathrm{GV}$ GCRs for different tilt angles $(\alpha=0,5,10$, 15, 30, 45, 60, and 75 degrees). Trajectories are traced back from Earth's position in different viewing directions. The daily harmonics are calculated for each declination separately. For brevity, 
we do not include the $23^{\circ}$ inclination of the Earth's rotation axis (which would introduce a seasonal variation). Trajectories are traced until they reach $90 \mathrm{AU}$. We find that the anisotropies are generated primarily in the inner heliosphere and they are insensitive to the precise location of the outer boundary. The observer (Earth) is placed in the helioequatorial plane and central symmetry is assumed w.r.t. the Sun. Hence the ecliptic components, $\xi_{\|}$and $\xi_{\perp}$ should be the same, while the North-South component, $\xi_{N S}$, should be the opposite in the two sectors. The results shown here always refer to times when the Earth is above the HCS in the Northern sector. Northern and Southern results would be interchanged in the opposite sector. The rigidity $100 \mathrm{GV}$ is chosen since it demonstrates basic features: regular motion dominates but GCRs are still substantially affected by the HMF. A future quantitative work shall cover a wider range of energy.
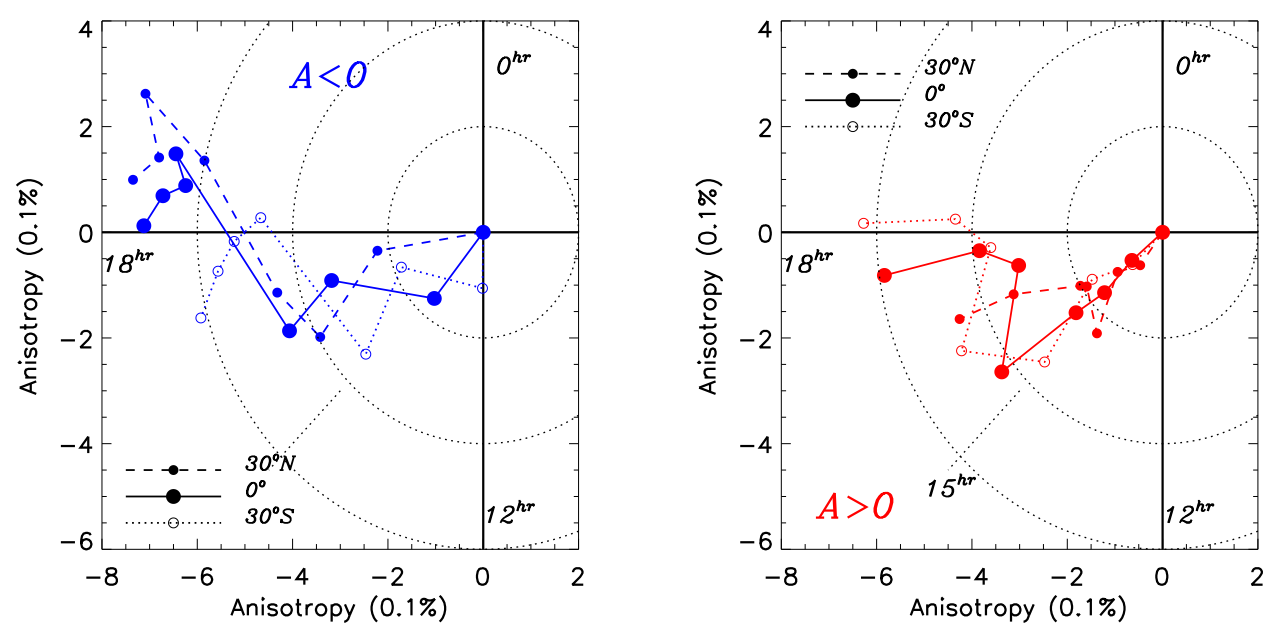

Figure 5: Simulation results for the ecliptic component of $100 \mathrm{GV}$ anisotropies. Blue/red dots refer to negative/positive polarity states. Dots indicate anisotropies starting from the origo in steps of increasing tilt angles: 0, 5, 10, 15, 30, 45, 60, and 75 degrees. Connecting lines are intended to guide the eye. Larger dots with solid line refer to equatorial, smaller full/open circles with dashed/dotted line refer to $30^{\circ} \mathrm{N}$ and $30^{\circ} \mathrm{S}$ directions, respectively (see text).

Figure 5 depicts the ecliptic components of the anisotropy predicted by the model of tracking pseudo-trajectories for tilt angles between $0^{\circ}$ and $75^{\circ}$ for the two polarity states $A<0$ (blue) and $A>0$ (red). The daily vectors are calculated for three declination separately: beside the equatorial directions (solid lines) also shown are predictions for the declinations $30^{\circ} \mathrm{N}$ (dashed) and $30^{\circ} \mathrm{S}$ (dotted). These three curves ought to be identical (except for a $\cos (\delta)$ geometrical factor) if the distribution of GCRs were produced by a pure first spharical harmonics. The systematic deviation of these curves from the symmetry (apart from statistical scatter) suggests that the directional distribution of GCRs is more complex and has substantial higher harmonics.

The idealized perfectly flat current sheet leads to isotropic distributions, hence curves start from the origo for both polarities, and increase with increasing tilt angle. The $A>0$ vectors are small and have their phases near $15 \mathrm{hr}$ as long as the tilt, $\alpha$ remains below $30^{\circ}$, Then they increase and their phases move toward 18hr. Finally (not shown here) the $A>0$ and $A<0$ vectors should be identical at $\alpha=90^{\circ}$. These features are in qualitative agreement with observations [8]. 

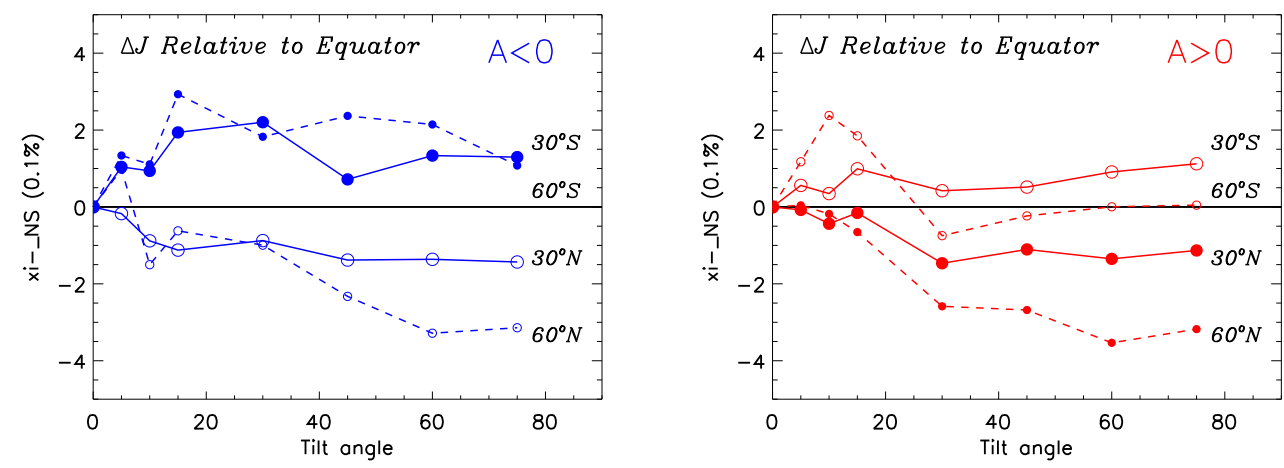

Figure 6: Average fluxes at 30 and 60, North and South declinations relative to the equatorial average (see text) for different tilt angles (same simulation as shown in Fig. 5). These are related to the N-S asymmetry through a $\sin (\delta)$ geometrical factor (see text).

As a measure of the (polarity dependent) North-South anisotropy, in Figure 6 we plot the daily averages of GCR intensities at different declinations relative to their equatorial value i.e. $(J(\delta)-J(0)) / J(0))$. For a pure first spherical harmonic of the directional distribution Northern and Southern curves would be the mirror images of each other and their amplitudes would be proportional to $\sin (\delta)$. Inspection of Fig. 6 shows significant asymmetry suggesting, again, the presence of substantial higher harmonics.

A remarkable result is that the predicted North-South differences are found to be significantly larger than those obtained from the diffusive approach at lower rigidities. This prediction is in qualitative agreement with the observation that MD data give substantially larger North-South anisotropies than NMs [8] (see Fig. 1). This encouraging finding needs further study and confirmation.

All runs in this section were carried out with the addition of a meager random component to the spiral $\operatorname{HMF}(\delta B / B \approx 0.2)$ at $1 \mathrm{AU}$ at the poles). We found that predicted anisotropies are fairly insensitive to magnitude of fluctuations in this range. It seems likely, however, that large-scale variations in the structure of the HCS, and the presence of corotating interaction regions are more effective than small scale fluctuations. This needs to be explored in future work.

\section{Conclusions}

The anisotropies of cosmic rays furnish valuable information. The reconstruction of threedimensional structure of the directional distributions of GCRs can be helpful to infer the transport parameters at NM energies [10], [11]. These considerations can be based on the diffusive equation of Parker [9].

Muon Detectors are sensitive to higher rigidities and can be used as probes of large-scale structures in the Heliosphere. At rigidities above 50GV, however, the gyro-radius of GCRs becomes sufficiently large (comparable to the heliocentric radius) and the diffusive model becomes inaccurate. The diffusive description is based in the assumption that the scattering mean free path and the gyro-radius of GCRs is small relative to the characteristic scales of the heliosphere (e.g. heliocentric radius). This is no longer valid at high rigidities. 
The intensity variation of GCRs in the Heliosphere are connected, with a few expections, with energy changes experienced by GCRs on their path before reaching the observer. Because of the negative spectral exponent of GCRs, an energy loss results in lower flux, while energy gain results in a flux increase. In the present work we adopted a method of calculating energy changes along (pseudo) trajectories of GCRs, tracked backwards in different directions from the observer. The method is similar to that of [4], with the difference that the present model can accommodate an additional random component of the HMF. Illustrative examples were presented for $100 \mathrm{GV}$ rigidity. The intent of the work is to demonstrate how the method works and no effort was made to achieve quantitative fits.

We considered the general features seen in the 44 years worth of NM and MD data covering two full 22-year cycles. Some robust trends are clearly seen. The model calculations presented here are in qualitative agreement with several observations. The phase of the solar daily vector shifts to earlier hours in $A>0$ cycles and its amplitude decreases during low solar activity as the HCS flattens. We find it encouraging that the model also reproduces the qualitative features of the North-South anisotropy and the magnitude of $\xi_{N S}$ turns out to be substantially larger for MD than for NMs. This subtlety has posed a challenge for previous models.

\section{Acknowledgments}

This work was carried out by the joint research program of the Institute for Space-Earth Environmental Research, Nagoya University

\section{References}

[1] T. Thambyahpillai \& H. Elliot, Nature, 171 (1953) 918

[2] S.E. Forbush, J. Geophys. Res., 72 (1967) 4937

[3] E.H. Levy, J. Geophys. Res. 81 (1976) 2082

[4] G. Erdős \& J. Kóta, Astrophys. Space Sci., 67 (1980) 45

[5] M.S. Potgieter \& H. Moraal, Astrophys. J., 294 (1985) 425

[6] J. Kóta \& J.R. Jokipii, Adv. Space Res. bf 27 (2001) 607

[7] A. Gil, R. Modzelewska, \& M. Alania, Adv. Space Res. 50 (2012) 712

[8] K. Munakata et al., Astrophys. J., 791 (2014) 22

[9] E.N. Parker, Planet. Space Sci. 128 (1965) 664

[10] J.W. Bieber \& J. Chen, Astrophys. J., 372 (1991) 301

[11] J. Chen \& J.W. Bieber, Astrophys. J., 405 (1993) 375 